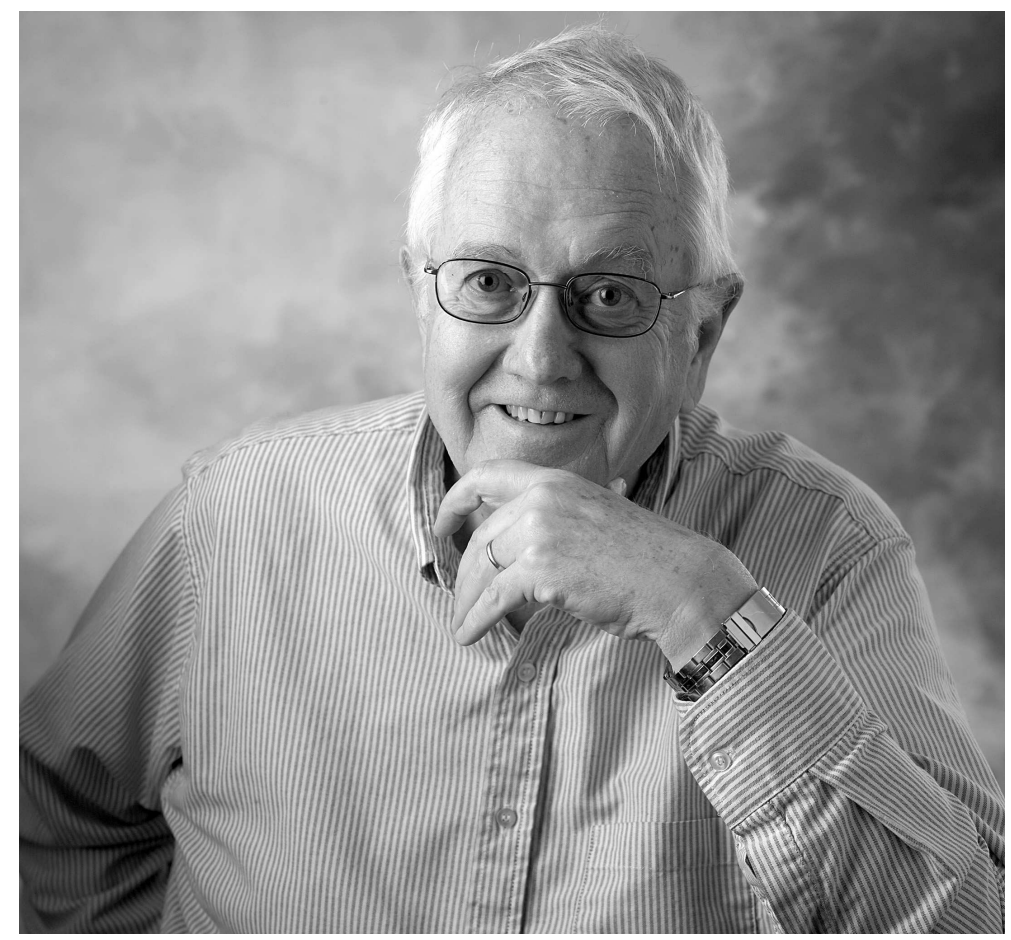

\title{
Douglas Henderson: from hard spheres to biological channels
}

Douglas Henderson belongs to a generation of physicists whose names are associated with the most recent progress in understanding of the liquid state of condensed matter. This progress was originated by studies of Kirkwood, followed by Wertheim's analytical solution of the Percus-Yevick equation for hard spheres and by a spreading this solution to the mean spherical approximation (MSA) for hard spheres with long range interaction by Waisman, Lebowitz, Blum, Stell and many others. These theoretical efforts were supported by an advance in computer simulation techniques that commenced with molecular dynamics and Metropolis Monte Carlo simulations by Alder and Wood. Doug's trace in this half a century play was summarized by George Jackson ${ }^{1}$ and is marked by early work in the 1960s on the virial expansions of correlation functions, his seminal work with John Barker on a perturbation theory of the liquid state in 1967, his exhaustive studies in the 1970s on prescriptions for the hard-sphere pair radial distribution function, the MSA solution of the Yukawa model, his developments for electrolyte solutions and metal electrodes, as well as his fundamental contribution to the integral equation techniques for inhomogeneous systems. In his 70 years, Doug Henderson has established a distinguished scientific career, has been a "named" professor at several universities throughout the world and has written over four hundred fifty papers. Some of the representative articles that have been pacesetting in our scientific community are listed hereinafter.

\footnotetext{
${ }^{1}$ Molecular Physics, 1995, vol. 86, No. 4
} 
It is not the purpose of this brief introduction to do justice to Doug's work. His life was celebrated ten years ago in a Festschrift in Molecular Physics. An important milestone since then was his 1999 Joel Hildebrand Award by the American Chemical Society in the Theoretical and Experimental Chemistry of Liquids sponsored by Exxon Research and Engineering Company and Exxon Chemical Company for "developing, with John Barker, the perturbation theory of liquids, the first successful theory of the liquid state, and for his continued development of the theory of liquids, liquid mixtures, liquid surfaces, and polarizable interfaces leading to significant advances in the theory of electrochemical interfaces and colloidal interactions". For the past five years, he has utilized simulations to explore the topics related to biological interfaces and inhomogeneous solutions with the same precision and care for which his mathematical approach is known. Doug has added simulations of ion channels and cavities in proteins to his range of heterogeneous solution interests. He worked very closely with us and with several other colleagues to examine the flow of ions through a neutral channel with nonequilibrium molecular dynamics, and then examined the origin of ion selectivity in sodium and calcium channels, defining the relative roles of charge neutralization and volume exclusion in the narrow cavities. Nowadays, he is trying to apply similar principles to the cavities associated with the active sites in such enzymes as HIV protease and acetylcholinesterase.

On August 7, 2004, the Henderson Symposium on Basic and Applied Statistical Mechanics of Condensed Matter was held at Brigham Young University in Provo, Utah. This Festschrift grew out of that occasion and includes some of the articles presented on that occasion, at which we also celebrated his 70th birthday. At the celebration banquet after the symposium, Farid Abraham, a long-time friend and colleague of Dr. Henderson, addressed the audience and gave some pleasant reflections on Doug's contributions. Because it was a simple and warm description of this wonderful man, we decided to use selections from his after-dinner speech as the introduction to the Festschrift. We join Farid Abraham in respect and admiration for this gentleman whom Peter Jordan, another colleague from the biological channel field, once referred to as "a class act".

\author{
Bob Eisenberg \\ David Busath \\ Andrij Trokhymchuk
}




\section{Some of papers of Douglas Henderson which he considers as most important}

- Barker J.A., Henderson D., Perturbation Theory and Equation of State for Fluids. II. A Successful Theory of Liquids. J. Chem. Physics, 47, December 1, 1967, 4714-4721.

- Smith W.R., Henderson D., Analytical Representation of the Percus-Yevick Hard-Sphere Radial Distribution Function. Molecular Physics, 19, September 1970, 411-415.

- Grundke E.W., Henderson D., Distribution Functions of Multicomponent Fluid Mixtures of Hard Spheres. Molecular Physics, 24, August 1972, 269-281.

- Barker J.A., Henderson D., What is 'Liquid'? Understanding the States of Matter. Reviews of Modern Physics, 48, October 1976, 587-671.

- Henderson D., Abraham F.F., Barker J.A., The Ornstein-Zernike Equation for a Fluid in Contact with a Surface. Molecular Physics, 31, April 1976, 12911295; reprinted 2002, 100, No. 1, 129-132.

- Henderson D., Blum L., Lebowitz J.L., An Exact Formula for the Contact Value of the Density Profile of a System of Charged Hard Spheres near a Charged Wall. Journal of Electroanalytical Chemistry and Interfacial Electrochemistry, 102, October 10, 1979, 315-319.

- Schmickler W., Henderson D., The Interphase Between Jellium and a Hard Sphere Electrolyte. A Model for the Electric Double Layer. Journal of Chemical Physics, 80, April 1, 1984, 3381-3386.

- Henderson D., Lozada-Cassou M., A Simple Theory for the Force Between Spheres Immersed in a Fluid. Journal of Colloid and Interface Science, 114, November 1986, 180-183.

- Plischke M., Henderson D., Pair Correlation Functions and Density Profiles in the Primitive Model of the Electric Double Layer. Journal of Chemical Physics, 88, February 15, 1988, 2712-2718.

- Henderson D., Sokolowski S., Hard Sphere Bridge Function Calculated From a Second-Order Percus-Yevick Approximation. Journal of Chemical Physics, 103, November 1, 1995, 7541-7544.

- Trokhymchuk A., Henderson D., Wasan D.T., A Molecular Theory of the Hydration Force in an Electrolyte Solution. Journal of Colloid and Interface Science, 210, February 15, 1999, 320-331.

- Boda D., Henderson D., Chan K.-Y., Monte Carlo Study of the Capacitance of the Double Layer in a Molten Salt. Journal of Chemical Physics, 110, March 15, 1999, 5346-5350. 
- Crozier P.S., Rowley R.L., Holladay N.B., Henderson D., Busath D.D., Molecular Dynamics Simulation of Continuous Current Flow Through a Model Biological Membrane Channel. Physical Review Letters, 86, March 12, 2001, $2467-2470$.

- Holovko M., Kapko V., Henderson D., Boda D., On the Influence of Ionic Association on the Capacitance of an Electric Double Layer. Chemical Physics Letters, 341, June 22, 2001, 363-368.

- Boda D., Gillespie D., Nonner W., Henderson D., Eisenberg B., Computing Induced Charges in Inhomogeneous Dielectric Media: Application in a Monte Carlo Simulation of Complex Ionic Systems. Phys. Rev. E, 69, April, 2004, article 046702, 1-10. 